\title{
Nach der Reform ist vor der Reform: Ungelöste Fragen der Gesundheitsreform 2007
}

\begin{abstract}
Die Gesundheitsreform der Großen Koalition ist verabschiedet und Teile davon sind zum April 2007 in Kraft getreten. Mit dieser Reform geht zwar eine Reihe institutioneller Neuerungen für das deutsche Gesundheitssystem einher. Zahlreiche Schwachstellen des Krankenversicherungssystems, die im Vorfeld des Reformprozesses als Kernprobleme diskutiert worden sind, bleiben dadurch jedoch unangetastet. Auch nach dem Gesundheitskompromiss wird und sollte das Thema Weiterentwicklung des Gesundheitswesens daher auf der politischen Agenda bleiben. Dieser Beitrag verortet die jüngste Gesundheitsreform im Kontext vergangener Reformziele und Gesundheitsreformen in Deutschland und zeigt auf, welche Problempunkte auch in Zukunft ungelöst und weiterentwicklungsbedürftig sind.
\end{abstract}

\section{GESUNDHEITSPOLITISCHE ZIELE UND REFORMPFADE VOR 2007}

Ähnlich wie für die Volkswirtschaft lässt sich für die Gesundheitspolitik ein „magisches Viereck" konkurrierender Ziele aufzeigen, die nicht gleichzeitig optimierbar sind: ${ }^{1}$

- Qualität: Gewährleistung einer hochwertigen Gesundheitsversorgung;

- Solidarität: gleichwertige Versorgung der Bevölkerung mit Gesundheitsleistungen, die unabhängig vom Einkommen der Versicherten ist;

- Wachstum: Sicherung und Schaffung von Arbeitsplätzen sowie Gewinnmöglichkeiten für die Leistungsanbieter im Gesundheitsmarkt;

- Finanzierbarkeit: Stabilisierung und nachhaltige Entwicklung der Gesundheitsausgaben.
Die Entwicklung des deutschen Gesundheitssystems bis vor der Reform 2007 lässt sich in drei Phasen unterteilen, in denen jeweils unterschiedliche Gewichtungen dieser vier Ziele erkennbar sind.

In der ersten Phase bis ca. 1975 (von Jens Alber als Phase der „doppelten Inklusion “ benannt) zeichnete sich die Entwicklung der Gesetzlichen Krankenversicherung (GKV) durch die fortschreitende Integration weiterer Bevölkerungsgruppen sowie die Ausweitung des Leistungskataloges aus. In dieser Phase standen vor allem die Ziele der Solidarität und des Wachstums im Zentrum.

Die zweite Phase von 1975 bis Anfang der 1990er Jahre lässt sich als Periode der traditionellen Kostendämpfungspolitik zulasten des Solidaritätszieles - bezeichnen. Die Ausweitung des GKV-Systems geriet Mitte der 1970er Jahre mit dem Ende der Nachkriegsprosperität ins Stocken. Daher wurde versucht, mit Kostendämpfungsgesetzen die Ausgabenzuwächse der GKV einzugrenzen. Leitlinie der Gesundheitspolitik war seitdem die sogenannte „einnahmeorientierte Ausgabenpolitik“, bei der die Ausgaben nicht stärker ansteigen sollten als die Einnahmen. Der Beitragssatzstabilität - und damit dem Ziel der nachhaltigen Finanzierbarkeit - wurde ein zentraler Stellenwert eingeräumt. Es kam zu ersten Privatisierungstendenzen in Form von individuellen Zuzahlungen sowie gewissen Leistungsausgrenzungen. Die bis dahin entstandenen Steuerungsstrukturen des Systems - z.B. die starke Bedeutung korporatistischer Verhandlungsstrukturen und der sozialen Selbstverwaltung oder das Vertragsmonopol der Kassenärztlichen Vereinigungen (KV) - und seine spezifischen Anreizstrukturen für die involvierten Akteure blieben jedoch weitgehend unberührt.
Dies änderte sich mit dem Gesundheitsstrukturgesetz (GSG) von 1992 und den nachfolgenden Reformen. Seit Anfang der 1990er Jahre, dem Beginn der dritten Phase, setzte sich zwar die Politik der Kostendämpfung fort. Im Gegensatz zum vorangegangenen Zeitraum blieben nun die traditionellen Versorgungs- und Finanzierungsstrukturen jedoch nicht mehr unangetastet. Dazu zählen beispielsweise die Neuorganisation der Krankenkassenordnung durch freie Kassenwahl und Risikostrukturausgleich, die Einführung von Budgetierungen im ambulanten und stationären Sektor sowie erweiterte Möglichkeiten für die Kassen, mit einzelnen Gruppen von Ärzten oder einzelnen Krankenhäusern - und nicht mehr ausschließlich mit den KV - Verträge zu schließen. Diese Phase zeichnet sich durch ein häufiges Wechselspiel der eingangs genannten Ziele aus. Mit dem GSG setzte sich infolge des „Lahnsteiner Kompromisses" zwischen der christlich-liberalen Bundestagsmehrheit und dem sozialdemokratisch geführten Bundesrat eine informelle Große Koalition erstmals mit weitreichenden Strukturreformen und Kostendämpfungsmaßnah-

\footnotetext{
1 Bandelow, N. (2006): Gesundheitspolitik: Zielkonflikte und Politikwechsel trotz Blockaden, in: Schmidt, M. G./ Zohlnhöfer, R. (Hrsg.): Regieren in der Bundesrepublik Deutschland. Innen- und Außenpolitik seit 1949, Wiesbaden, S. 159.
}

Simone Leiber, Dr., ist Wissenschaftlerin im WSI in der Hans-Böckler-Stiftung. Arbeitsschwerpunkte: Europäische Sozialpolitik, Wohlfahrtsstaaten im Vergleich, Gesundheitspolitik.

e-mail: Simone-Leiber@boeckler.de 
men gegen die traditionell einflussreichen Anbieterinteressen des Gesundheitssystems durch. So wurden z. B. Pharmaunternehmen zu Preissenkungen für patentgeschützte Arzneimittel gezwungen. Die Wachstumsinteressen der Anbieter wurden in dieser Phase weniger stark gewichtet als zuvor.

In den Folgejahren setzten sich Kostendämpfungsmaßnahmen unter der schwarz-gelben Regierung fort. Da diese nicht mehr im Konsens mit der Opposition verabschiedet wurden, wurden einige dieser Reformschritte zu Beginn der rotgrünen Regierung rückgängig gemacht (z.B. wurden Selbstbehalte gesenkt). Zeitweise wurde somit das Ziel der Solidarität wieder stärker in den Vordergrund gestellt. Das Gesundheitsmodernisierungsgesetz (GMG) 2003 ging dann aber mit einer ungekannt weitgehenden Umverteilung der Finanzierungslasten von den Arbeitgebern zu den Patienten und Versicherten einher (umfangreiche Zuzahlungen und Leistungsausgliederungen). Gleichzeitig zielte es auf eine Modernisierung der Versorgungsstrukturen und damit Verbesserung der Versorgungsqualität. Dazu zählen etwa Ansätze, die Koordinierungsfunktion des Hausarztes wiederzubeleben, sowie die Einrichtung von strukturierten Behandlungsprogrammen für chronisch Kranke (Disease Management Programme/DMP) oder die Aufwertung der Prävention.

\section{DER GESUNDHEITSKOMPROMISS 2007: (NICHT) ERREICHTE ZIELE}

Der Gesundheitskompromiss der Großen Koalition setzt sich aus vier Kernbereichen zusammen: die Einführung einer allgemeinen Versicherungspflicht; Reformen der Versorgungsstrukturen und Kassenorganisation; Reform der Finanzierungsordnung sowie Reformen in der privaten Krankenversicherung. Wie lassen sich die beschlossenen Reformen vor dem Hintergrund des eingangs beschriebenen Ziel-Quadrilemmas verorten?

\section{QUALITÄT DER VERSORGUNG}

Die Reformdiskussion im Vorfeld des Gesundheitskompromisses war vor allem von finanzpolitischen Erwägungen getragen. So gehörte etwa eine Weiterentwicklung des während der rot-grünen Regierung nicht zustande gekommenen Präventionsgesetzes nicht zum verhandelten Reform- paket. Dennoch beinhaltet der Kompromiss einige Ansätze zur Verbesserung der Versorgungsqualität. Dazu gehören etwa Maßnahmen, welche die Versorgung für Sterbende und Schwerstkranke (Palliativmedizin) ausbauen sollen, die Öffnung der Krankenhäuser für die ambulante Versorgung, ein Rechtsanspruch auf Rehabilitation sowie Impfungen und Kuren, die zu Pflichtleistungen werden. Des Weiteren sind die Krankenkassen seit April 2007 gehalten, eine größere Bandbreite an Wahltarifen anzubieten, die es den Versicherten ermöglichen soll, das Versorgungsangebot besser auf ihre individuellen Bedürfnisse zuzuschneiden. Bei der Beurteilung dieser Tarife, die häufig einheitlich unter den Stichwort „mehr Wahlfreiheit" angepriesen werden, muss jedoch beachtet werden, dass diese im Einzelnen sehr unterschiedliche Wirkungen entfalten. Während Tarife für eine hausarztzentrierte Versorgung, für von der Regelversorgung ausgeschlossene Therapien (z.B. Homöopathie) oder für die Teilnahme der Versicherten an besonderen Versorgungsformen wie DMP tatsächlich zu einer Optimierung der Versorgungsqualität beitragen können, haben Selbstbehaltund Kostenerstattungstarife sowie Tarife für die Nichtinanspruchnahme von Leistungen vor allem einen starken Entsolidarisierungseffekt. Experten erwarten zudem negative Folgen des über den Gesundheitsfonds initiierten Kassenwettbewerbs für die Versorgungsqualität, unter anderem, da im Reformwerk angelegte Wettbewerbsverzerrungen trotz vielfältiger Kritik von Verbänden und aus der Wissenschaft nicht korrigiert wurden. So wirkt die im Rahmen des Gesundheitsfonds eingeführte individuelle Überforderungsklausel ${ }^{2}$ - obwohl aus sozialpolitischen Gesichtspunkten sinnvoll zulasten von Krankenkassen mit vielen einkommensschwachen Patientinnen und Patienten: „Dass eine Krankenkasse mit Defiziten die Möglichkeiten, Zeit und Vertragsmacht hat, für ihre Versicherten nur nicht-effektive Leistungen zu kappen, ist illusorisch."3

\section{SOLIDARITÄT}

Hinsichtlich des Solidaritätszieles lassen sich die Ergebnisse des Gesundheitskompromisses wie folgt zusammenfassen: Die Einführung einer allgemeinen Versicherungspflicht, gekoppelt mit Hilfestellungen für diejenigen, die ihren Versicherungsschutz verloren haben und/oder sich die
Versicherungstarife nicht mehr leisten können, erhöht den Solidarcharakter des deutschen Gesundheitssystems. Erreicht wurde vor allem eine gewisse Stärkung solidarischer Elemente innerhalb der Privaten Krankenversicherung (PKV). Die Solidarbeziehungen zwischen GKV und PKV blieben dagegen unangetastet, während man innerhalb der GKV aufgrund der genannten Wahltarife sogar von einem Entsolidarisierungsprozess sprechen kann.

Die neuen PKV-Basistarife mit Kontrahierungszwang führen dazu, dass auch in der PKV künftig niemand mehr aufgrund einer schlechten Gesundheit abgewiesen werden kann und dass auch eine private Versicherung für Menschen mit schlechten Gesundheitsrisiken bezahlbar bleibt. Der Basistarif muss in seinem Leistungsumfang der GKV vergleichbar sein und darf den GKV-Höchstbetrag nicht überschreiten. Wenn die Versicherungsprämie Hilfebedürftigkeit (d.h. Inanspruchnahme der Grundsicherung für Arbeitsuchende oder Sozialgeld) auslösen würde, sind künftig staatliche Zuschüsse vorgesehen.

Während PKV-Neuversicherte ein Wechselrecht in den Basistarif jedes beliebigen PKV-Unternehmens erhalten, hat man allerdings die Wechselmöglichkeiten für PKV-Altkunden in der letzten Verhandlungsphase des Reformprozesses auf den Zeitraum vom 1.1.2009 bis 30.6.2009 beschränkt. Danach können diese nur noch in den Basistarif ihrer eigenen PKV übertreten und dies auch nur, wenn es sich um Bedürftige oder über 55-Jährige handelt. Die etwa acht Millionen privat Versicherten müssen Anfang 2009 somit eine nicht korrigierbare Entscheidung über ihren künftigen Versicherungsschutz treffen. Dabei können sie zu diesem Zeitpunkt voraussichtlich nur schwer absehen, welches Unternehmen dauerhaft das beste Angebot leisten wird. Auch die - grundsätzlich positive - Verbesserung der Übertragbarkeit von Altersrückstellungen bei einem Wechsel des Versicherers blieb hinter den ursprünglich diskutierten Plänen zurück

\footnotetext{
2 Diese sieht vor, dass die im Rahmen des Fonds ermöglichten "kleinen Kopfpauschalen", wenn sie über $8 €$ liegen, nicht mehr als $1 \%$ des beitragspflichtigen Einkommens der Versicherten ausmachen dürfen.

3 Lüngen, M./Gerber, A./Lauterbach, K. W. (2006) Gesundheitsfonds oder/und steuerfinanziert - die Umgestaltung der Krankenversicherung, in: ifo Schnelldienst 16, S. 4.
} 
und findet nun nicht in vollem, sondern lediglich im Umfang des Basistarifs statt.

Insgesamt führen diese Reformschritte dazu, das „Rosinenpicken“ der PKV in Bezug auf Versicherte mit guten Gesundheitsrisiken etwas einzuschränken. Die grundlegenden Ungerechtigkeiten, Wettbewerbsverzerrungen und die geschlechterpolitische Schieflage der in PKV und GKV gespaltenen Vollversicherung in Deutschland ${ }^{4}-$ obwohl in dieser Weise einmalig in Europa und im Vorfeld der Reformdiskussion von wissenschaftlicher Seite einhellig kritisiert - wurden jedoch nicht aufgelöst.

Entsolidarisierung in der GKV erfolgte in diesem Reformprozess auf anderem Wege als bei vorangegangenen Reformen, bei denen vor allem individuelle Zuzahlungen der Versicherten und Leistungsausgliederungen im Zentrum standen. Zu weiteren Ausgliederungen aus dem Leistungskatalog kam es in dieser Reformphase - bis auf wenige Ausnahmen im Bereich sogenannter selbstverschuldeter Behandlungsbedürftigkeit (z. B. Schönheitsoperationen, Piercing, Tätowierungen) - nicht. Eine erneute Verlagerung von Kosten auf die Schultern der Versicherten ist jedoch von den „kleinen Kopfpauschalen“ im Rahmen des neu eingeführten Gesundheitsfonds zu erwarten. Hinzu kommt: Selbstbehalt-, Kostenerstattungstarife und Tarife für die Nichtinanspruchnahme von Leistungen sind vor allem für Versicherte mit guten Risiken bzw. hinreichendem Einkommen, das als „Sicherheitspolster“ auch bei höher ausfallenden Behandlungskosten dient, attraktiv. Für Versicherte mit schlechten Risiken und/oder sehr niedrigem Einkommen besteht eine Wahlfreiheit bezüglich dieser Tarife faktisch nicht. Ursprünglich wurde deren Einführung damit begründet, eine Abwanderung guter Risiken in die PKV zu verhindern. Statt die ungleichen Wettbewerbsbedingungen zwischen PKV und GKV abzuschaffen, hat man nun jedoch neue Ungleichheiten innerhalb der Gruppe der GKV-Versicherten geschaffen.

\section{FINANZIERBARKEIT UND WACHSTUM}

In Bezug auf das Ziel einer nachhaltigen Sicherung der Finanzierbarkeit des Gesundheitswesens bleiben Ergebnisse des Koalitionskompromisses am stärksten hinter den ursprünglich formulierten Ansprüchen der Politik zurück. Viele Experten sind sich darin einig: Will man das Ge- sundheitssystem langfristig stabil ausrichten, führt kaum ein Weg an einer stärkeren Steuerfinanzierung der GKV vorbei. Die Finanzprobleme in der GKV resultieren vor allem aus der im Vergleich zur Entwicklung des Bruttoinlandsproduktes (BIP) bzw. der Ausgaben deutlich unterdurchschnittlichen Entwicklung der Einnahmenbasis, nämlich der Bruttolohn- und gehaltssumme (BLG). In Zeiten einer anhaltend schwachen Lohn- und Beschäftigungsentwicklung bereitet die Wahl der BLG als Bezugsbasis für die Einnahmen große Probleme. Hätte man an Stelle der BLG eine breite Steuerbasis als Bezugsgröße für die Einnahmen gewählt, dann hätte eine realistische Chance bestanden, dass die GKV nicht so rasch in Finanzprobleme läuft. Eine stärkere Steuerfinanzierung erweitert zudem automatisch den Kreis der an der Finanzierung Beteiligten, z.B. auch auf Beamte und Selbstständige, und es erweitert sich die Bemessungsgrundlage um zusätzliche Einkommensarten (Gewinne, Zinsen, Mieten, Einnahmen der Selbstständigen). Alternativ hätte eine substanzielle Stärkung der Einnahmebasis auch auf dem Wege einer Bürgerversicherung (verstanden als Einbeziehung aller Bürger in ein gemeinsames Versicherungssystem unter Verbeitragung zusätzlicher Einkunftsarten) erreicht werden können. Der Gesundheitskompromiss sieht jedoch weder diese Schritte in Richtung Bürgerversicherung noch eine gesicherte Erhöhung des Steuerzuschusses zur GKV vor. Im Gegenteil: Das Auslaufen des Zuschusses aus der Tabaksteuer von 4,2 Mrd. $€$ bis 2008 wird durch die geplante Steuerfinanzierung der Kinderversicherung in der GKV für diesen Zeitraum (2,5 Mrd. € im Jahr 2008) nicht einmal kompensiert. Für die in den Folgejahren geplante weitere Anhebung des steuerlichen Kinderzuschusses (um jährlich 1,5 bis auf $14 \mathrm{Mrd}$. €) wurde die Gegenfinanzierung noch nicht geklärt. Die Einführung des Gesundheitsfonds ohne eine Einbeziehung der PKV und ohne einen substanziellen Umstieg auf eine Steuerfinanzierung - ändert an dieser Situation nichts. Unter den gegebenen Umständen ist also mit einem chronisch unterfinanzierten Fonds und somit mit einer Privatisierung von Kosten für die Versicherten in Form von „kleinen Kopfpauschalen" zu rechnen. Die moderat umgesetzte Steuerfinanzierung hat ebenfalls zur Folge, dass mit dem Start des Fonds von einer Steigerung der dann vereinheitlichten und staatlich festgelegten Beitragsätze von mindestens 0,5\%-Punkten im Vergleich zum heutigen Durchschnittssatz gerechnet wird. Auch bezüglich des Wachstumszieles bzw. der erwartbaren Auswirkungen auf die Arbeitsplatzsituation sind vom Gesundheitskompromiss also eher bremsende Effekte zu erwarten.

Auf der Ausgabenseite wurden einige sinnvolle Kostendämpfungsmaßnahmen vorgenommen und beispielsweise eine Kosten-Nutzen-Bewertung für Arzneimittel eingeführt. Auch sieht der Kompromiss eine komplette Neuorientierung der Vergütung für den ambulanten ärztlichen Bereich vor. Dass diese Umstellung, bei der das Morbiditätsrisiko von den Arztpraxen auf die Krankenkassen übergeht, zu Einsparungen führt, wird allerdings bezweifelt. ${ }^{5}$ Auch in diesem Feld wären durchaus weitergehende Schritte denkbar gewesen, die einen qualitätsorientierten Wettbewerb auch auf der Seite der Leistungserbringer in Gang setzen.

\section{KÜNFTIGE HERAUSFORDERUNGEN}

Der Blick auf den Gesundheitskompromiss 2007 anhand des Ziel-Quadrilemmas ergibt eine ernüchternde Bilanz: Abgesehen von einigen positiven Ansätzen im Bereich der Versorgungsqualität wurde keines der vier Ziele wesentlich optimiert. Insbesondere vom Ziel der nachhaltigen Finanzierbarkeit, das ursprünglich im Vordergrund der Reformdiskussion stand, ist man besonders weit entfernt geblieben. Es bleibt also auch in Zukunft die große Herausforderung bestehen, im Spannungsfeld der vier Ziele möglichst weitgehende Fortschritte in möglichst vielen Bereichen zu erzielen. Wird diese Herausforderung weiter auf die lange Bank geschoben, besteht die Gefahr, dass das Spannungsverhältnis der vier Ziele letztlich noch weiter zulasten der Solidarität aufgelöst wird. Noch immer gibt es Umfragen zufolge derzeit in der Bevölkerung ein hohes Maß an Unterstützung für eine solidarische Organisation der Krankenversicherung und auch die Bereit-

\footnotetext{
4 Ausführlich dazu z. B. Leiber, S./Zwiener, R. (2006): Zwischen Bürgerversicherung und Kopfpauschale: Vorschläge für eine tragfähige Kompromisslösung WSI-Diskussionspapier 146

5 Z. B. Kiefer, G./Ruiss, D. (2006): Staatliche, administrierte Gesundheitsversorgung - Die Eckpunke der Großen Koalition zur Gesundheitsreform, in: Sozialer Fortschritt 16, S. 259.
} 
schaft, eine höhere Abgabenbelastung dafür in Kauf zu nehmen, solange eine gute Versorgungsqualität gewährleistet ist. In Zeiten enger werdender Verteilungsspielräume gewinnt jedoch die Tatsache an Bedeutung, dass Umverteilung transparenten, konsistenten und gerechten Regeln folgt. Ist dies nicht der Fall, könnte das mittelfristig mit einer Aushöhlung der Akzep$\tan z$ des Solidarprinzips verbunden sein. Künftige Reformen stehen also dringlich vor der Aufgabe, die aufgezeigten Bruch- stellen des Solidarprinzips im deutschen Krankenversicherungssystem anzugehen und die Versorgungsqualität weiter zu verbessern. Dies gilt im Übrigen im selben Maße für die Pflegeversicherung. Über eine substanzielle Umfinanzierung von Beiträgen auf Steuermittel wäre zudem ein Weg gefunden, der eine nachhaltigere Finanzierung erlauben würde, ohne dem Wachstumsziel zwangsläufig entgegenzustehen. Ein solches solidarisches Reformkonzept schließt den Rückgriff auf Wettbe- werbsprinzipien nicht aus. Allerdings darf der Wettbewerb nicht selektiv nur für die Leistungsträger des Gesundheitswesens eingesetzt werden, sondern muss insbesondere auf der Seite der Leistungserbringer ebenfalls konsequent weiterentwickelt werden. Quasi wettbewerbsfreie Zonen wie das deutsche Apothekensystem, das insbesondere von den politischen Kräften verteidigt wird, die in anderen Feldern laut nach Marktprinzipien rufen, sind dann nicht mehr adäquat.

\section{Tarifbindung und betriebliche Interessen- vertretung - Aktuelle Ergebnisse aus dem IAB-Betriebspanel 2006}

Das in Deutschland praktizierte System der Lohnfindung und der Arbeitsbeziehungen steht seit Jahren in der Diskussion. Die Situation ist heute in West- wie Ostdeutschland durch eine wesentlich größere Differenzierung und Dezentralisierung gekennzeichnet als in den 1990er Jahren. Bis dahin wurden Löhne und Arbeitsbedingungen in Westdeutschland überwiegend branchenweit geregelt. Die dominierende Rolle des branchenweiten Verbandstarifvertrages und der ihn schließenden Tarifparteien hat seither abgenommen, betriebsbezogene Regelungen und die betriebliche Interessenvertretung haben an Bedeutung gewonnen, und das gesamte Lohnfindungssystem ist vielschichtiger geworden.

Die einzige repräsentative Datenquelle, die Ergebnisse zur Tarifbindung und der betrieblichen Interessenvertretung für die Gesamtwirtschaft liefert, ist das IAB-Betriebspanel. Es enthält für Westdeutschland seit 1993 Informationen zur Existenz eines Betriebsrats, die Tarifbindung der Betriebe wird seit 1995 erfragt. 1996 wurden auch ostdeutsche Betriebe mit in die Erhebung einbezogen. Die Ergebnisse für 2006 beruhen auf den Angaben von rund 15.500 westund ostdeutschen Betrieben. Aufgrund des Aufbaus der Zufallsstichprobe sind die Ergebnisse repräsentativ für die rund zwei Mil- lionen Betriebe mit mindestens einem sozialversicherungspflichtig Beschäftigten. Insgesamt sind in diesen Betrieben 32,4 Millionen Personen beschäftigt.

\section{AUSMAß UND ENTWICKLUNG DER TARIFBINDUNG}

In Deutschland spielen überbetriebliche Verbands- oder Flächentarifverträge, die zwischen Arbeitgeberverbänden und Gewerkschaften branchenweit ausgehandelt werden, immer noch eine wesentliche Rolle. Löhne und Arbeitsbedingungen können aber auch auf Betriebs- oder Unternehmensebene (Firmentarifverträge) oder in individuellen Arbeitsverträgen geregelt werden. Die besondere Bedeutung tarifvertraglicher Regelungen im deutschen Arbeitsrecht kommt in ihrem Vorrang gegenüber Betriebsvereinbarungen und Einzelarbeitsverträgen zum Ausdruck. Regelungen in Tarifverträgen setzen eine Norm, die nicht unterschritten werden darf. So können sie auch als Mindestarbeitsbedingungen interpretiert werden.

Im Folgenden werden aktuelle Auswertungen des IAB-Betriebspanels für das Jahr 2006 dargestellt, wobei zwischen der Tarifbindung der Betriebe und der Beschäftigten unterschieden wird. Betrachtet man zunächst die Beschäftigten, so haben im Jahr 2006 hochgerechnet rund $57 \%$ der westdeutschen und etwa $41 \%$ der ostdeutschen Beschäftigten in einem Betrieb gearbeitet, der einem Branchentarifvertrag unterlag (Tabelle 1). Firmentarifverträge galten für $8 \%$ der westdeutschen und $13 \%$ der ostdeutschen Beschäftigten. Für gut ein Drittel der westdeutschen und $46 \%$ der ostdeutschen Arbeitnehmer gab es keinen Tarifvertrag. Jeweils rund die Hälfte dieser Arbeitnehmer wurde jedoch indirekt von Tarifverträgen erfasst, da sich ihre Betriebe

Peter Ellguth ist wissenschaftlicher Mitarbeiter im Institut für Arbeitsmarkt- und Berufsforschung (IAB) der Bundesagentur für Arbeit in der Projektgruppe IAB-Betriebspanel. Arbeitsschwerpunkte: Arbeitsbeziehungen und betriebliche Arbeitszeitpolitik. e-mail: peter.ellguth@iab.de Susanne Kohaut, Dr., ist wissenschaftliche Mitarbeiterin im Institut für Arbeitsmarktund Berufsforschung (IAB) der Bundesagentur für Arbeit in der Projektgruppe IAB-Betriebspanel.

Arbeitsschwerpunkte: Tarifbindung, Neugründen und betriebliche Innovationen. e-mail: susanne.kohaut@iab.de 
daran orientierten. Das Ausmaß der Tarifbindung variiert zwischen den Wirtschaftszweigen stark, wobei sich die Bedeutung für die einzelnen Branchen von Jahr $\mathrm{zu}$ Jahr wenig ändert.

Aufgrund der starken Größenabhängigkeit der Tarifbindung ergibt sich auf der Ebene der Betriebe, dass hochgerechnet rund $37 \%$ der westdeutschen, aber nur $20 \%$ der ostdeutschen Betriebe durch Branchentarifverträge gebunden waren. Haus- oder Firmentarifverträge galten für $3 \%$ der Betriebe in den alten und etwa $4 \%$ der Betriebe in den neuen Bundesländern (Tabelle 2). Der Rest, also etwa $61 \%$ der westdeutschen und drei Viertel der ostdeutschen Betriebe, war nicht tarifgebunden. Allerdings gaben etwa $40 \%$ der nicht tarifgebundenen Betriebe an, sich in ihren Einzelarbeitsverträgen an bestehenden Branchentarifen zu orientieren.

Betrachtet man die Tarifbindung der Betriebe nach ihrer Größe, so zeigt sich wie in den Vorjahren, dass der Deckungsgrad mit zunehmender Betriebsgröße steigt. Das gilt sowohl für die alten als auch für die neuen Bundesländer, wenn auch auf unterschiedlichem Niveau. Während für die Kleinbetriebe die Branchentarifverträge eine untergeordnete Rolle spielen, sind Großbetriebe mit über 500 Beschäftigten in der Mehrheit tarifgebunden. Ebenso nimmt die Bedeutung der Haus- bzw. Firmentarifverträge mit steigender Betriebsgröße zu.

Im IAB-Betriebspanel werden die Daten zur Tarifbindung seit 1996 für beide Landesteile erhoben. Die Flächentarifbindung zeigt in den alten wie in den neuen Bundesländern seither rückläufige Tendenz (Abbildung 1). In Westdeutschland ging die Flächentarifbindung bezogen auf die Beschäftigten von 1996 bis 2006 um 12 Prozentpunkte zurück. In Ostdeutschland belief sich dieser Rückgang sogar auf 15 Prozentpunkte. Bezogen auf die Betriebe betrugen die entsprechenden Rückgänge der Flächentarifbindung im Westen etwa 11 und im Osten rund 9 Prozentpunkte. Wie es sich bereits in den Vorjahren abzeichnete, scheint sich nun die Tarifbindung in Ostdeutschland auf einem niedrigeren Niveau zu stabilisieren.

Fasst man die beiden Landesteile zusammen, so gilt derzeit in Deutschland für etwa $34 \%$ der Betriebe mit rund $54 \%$ der Beschäftigten ein Branchentarifvertrag. Rund ein Viertel aller Betriebe orientiert sich darüber hinaus an einem Branchentarifvertrag, sodass weitere $19 \%$ der Be-

\begin{tabular}{|c|c|c|c|c|c|c|}
\hline \multirow[t]{2}{*}{ Branche } & \multicolumn{2}{|c|}{$\begin{array}{l}\text { Branchen- } \\
\text { tarifvertrag }\end{array}$} & \multicolumn{2}{|c|}{$\begin{array}{l}\text { Firmen- } \\
\text { tarifvertrag }\end{array}$} & \multicolumn{2}{|c|}{$\begin{array}{c}\text { Kein Tarifvertrag } \\
\text { (davon Orientierung } \\
\text { an einem Tarifvertrag) }\end{array}$} \\
\hline & West & Ost & West & Ost & West & Ost \\
\hline Landwirtschaft u.a. & 55 & 14 & * & $*$ & $42(31)$ & $85(46)$ \\
\hline Bergbau/Energie & 77 & 48 & 14 & 40 & $10(60)$ & $12(44)$ \\
\hline $\begin{array}{l}\text { Grundstoffverarbeitung } \\
\text { (Produktionsgüter) }\end{array}$ & 59 & 37 & 12 & 11 & $29(64)$ & $52(55)$ \\
\hline Investitionsgüter & 59 & 22 & 10 & 17 & $31(68)$ & $61(53)$ \\
\hline Verbrauchsgüter & 58 & 31 & 9 & 12 & $34(61)$ & $58(57)$ \\
\hline Baugewerbe & 73 & 48 & 3 & 7 & $23(70)$ & $46(65)$ \\
\hline Handel/Reparatur & 50 & 31 & 6 & 6 & $44(56)$ & $63(47)$ \\
\hline Verkehr/Nachrichten & 44 & 24 & 15 & 32 & $41(44)$ & $45(30)$ \\
\hline Kredit/Versicherung & 85 & 72 & 2 & * & $13(58)$ & $26(*)$ \\
\hline Dienste für Unternehmen & 35 & 42 & 8 & 7 & $57(30)$ & $51(40)$ \\
\hline sonstige Dienste & 56 & 38 & 8 & 15 & $36(51)$ & $46(49)$ \\
\hline Org. ohne Erwerbszweck & 53 & 30 & 7 & 28 & $40(71)$ & $42(35)$ \\
\hline Öffentliche Verwaltung & 87 & 84 & 10 & 14 & $4(78)$ & $3(*)$ \\
\hline Beschäftigte gesamt & 57 & 41 & 8 & 13 & $35(51)$ & $46(48)$ \\
\hline
\end{tabular}

Tabelle 2: Tarifbindung der Betriebe in West- und Ostdeutschland 2006 nach Branchen - in \% -

\begin{tabular}{lcccccc}
\hline & \multicolumn{2}{c}{$\begin{array}{c}\text { Branchen- } \\
\text { tarifvertrag }\end{array}$} & \multicolumn{2}{c}{$\begin{array}{c}\text { Firmen- } \\
\text { tarifvertrag }\end{array}$} & \multicolumn{2}{c}{$\begin{array}{c}\text { Kein Tarifvertrag } \\
\text { (davon Orientierung } \\
\text { an einem Tarifvertrag) }\end{array}$} \\
& West & Ost & West & Ost & West & Ost \\
\hline Landwirtschaft u.a. & 40 & 7 & $*$ & $*$ & $58(26)$ & $93(34)$ \\
Bergbau/Energie & 63 & 37 & 4 & 11 & $33(54)$ & $52(58)$ \\
Grundstoffverarbeitung & 34 & 20 & 4 & 5 & $62(56)$ & $76(43)$ \\
(Produktionsgüter) & 37 & 14 & 2 & 6 & $61(45)$ & $80(48)$ \\
Investitionsgüter & 48 & 20 & 2 & 4 & $50(47)$ & $76(54)$ \\
Verbrauchsgüter & 60 & 40 & 2 & 3 & $38(60)$ & $57(62)$ \\
Baugewerbe & 35 & 17 & 2 & 5 & $63(45)$ & $78(36)$ \\
Handel/Reparatur & 30 & 15 & 6 & 4 & $64(46)$ & $81(32)$ \\
Verkehr/Nachrichten & 40 & 28 & 3 & $*$ & $58(41)$ & $69(17)$ \\
Kredit/Versicherung & 14 & 12 & 1 & 3 & $85(31)$ & $85(32)$ \\
Dienste für Unternehmen & 39 & 16 & 3 & 5 & $59(41)$ & $79(42)$ \\
sonstige Dienste & 46 & 24 & 7 & 7 & $47(45)$ & $69(38)$ \\
Org. ohne Erwerbszweck & 84 & 91 & 8 & 4 & $8(72)$ & $5(*)$ \\
Öffentliche Verwaltung & 37 & 20 & 3 & 4 & $61(42)$ & $75(40)$ \\
\hline Betriebe gesamt & & & & & & WSI MITTEILUNGEN
\end{tabular}

schäftigten zumindest indirekt von den Regelungen eines Tarifvertrages profitieren. Damit wird die immer noch dominierende Funktion des Flächentarifvertrags deutlich.

\section{AKTUELLE ERGEBNISSE ZUR BETRIEBLICHEN MITBESTIMMUNG}

Die Betrachtung der zweiten tragenden Säule im bundesdeutschen dualen System der Interessenvertretung - die Betriebsräte - ließ sich in den letzten Jahren immer mit den Worten „business as usual“ zusammenfassen. Inwieweit sich dies auch mit Blick auf die aktuellen Ergebnisse sagen lässt, wird im Folgenden kurz dargestellt. Zunächst soll analog zur Tarifbindung ein Eindruck der quantitativen Entwicklung der betrieblichen Mitbestimmung bis 2006 vermittelt werden. Dazu sind in Tabelle 3 Daten aus dem IAB-Betriebspanel zur Existenz von Betriebsräten in der Privatwirtschaft (Betriebe ab fünf Beschäftigte) seit 1993 für Westdeutschland und 1996 für Ostdeutschland dargestellt.

Für den Anteil der Betriebe mit gesetzlich legitimierter Interessenvertretung lässt sich weder in den alten noch den neuen Bundesländern ein eindeutiger Trend ausmachen. Die Anteilswerte schwanken um die 11\%-Marke. In Bezug auf den Anteil 


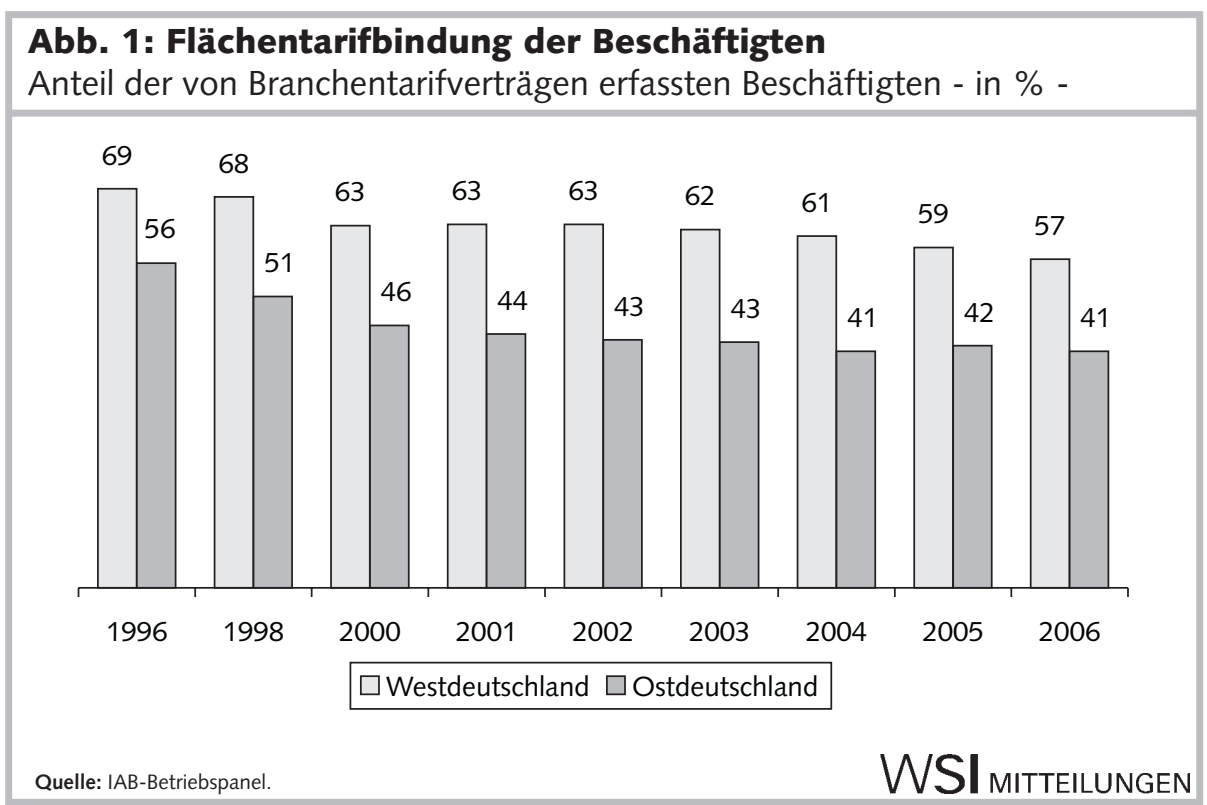

Tabelle 3: Betriebe bzw. Beschäftigte in Betrieben mit einem Betriebsrat 1993 bis 2006* - in \% -

\begin{tabular}{|c|c|c|}
\hline Jahr & $\begin{array}{l}\text { Anteil der Betriebe } \\
\text { mit Betriebsrat }\end{array}$ & $\begin{array}{l}\text { Anteil der Beschäftigten } \\
\text { in Betrieben mit Betriebsrat }\end{array}$ \\
\hline \multicolumn{3}{|c|}{ Westdeutschland } \\
\hline 1993 & 10 & 50 \\
\hline 1994 & 11 & 51 \\
\hline 1996 & 12 & 51 \\
\hline 1998 & 10 & 50 \\
\hline 2000 & 12 & 50 \\
\hline 2002 & 11 & 50 \\
\hline $2003 * *$ & 11 & 48 \\
\hline 2004 & 10 & 47 \\
\hline 2005 & 11 & 47 \\
\hline \multirow[t]{2}{*}{2006} & 10 & 46 \\
\hline & \multicolumn{2}{|c|}{ Ostdeutschland } \\
\hline 1996 & 10 & 42 \\
\hline 1998 & 10 & 38 \\
\hline 2000 & 12 & 40 \\
\hline 2002 & 11 & 40 \\
\hline $2003 * *$ & 11 & 39 \\
\hline 2004 & 10 & 38 \\
\hline 2005 & 11 & 39 \\
\hline 2006 & 10 & 38 \\
\hline \multicolumn{3}{|c|}{$\begin{array}{l}\text { *Basis: privatwirtschaftliche Betriebe ab } 5 \text { Beschäftigte, ohne Landwirtschaft und Organisationen ohne } \\
\text { Erwerbszweck. }\end{array}$} \\
\hline
\end{tabular}

\begin{tabular}{|c|c|c|c|c|c|c|}
\hline & & & Betriebsg & ßenklassen & & \\
\hline & $\begin{array}{l}5-50 \\
\text { Besch. }\end{array}$ & $\begin{array}{l}51-100 \\
\text { Besch. }\end{array}$ & $\begin{array}{c}\text { 101-199 } \\
\text { Besch. }\end{array}$ & $\begin{array}{c}200-500 \\
\text { Besch. }\end{array}$ & $\begin{array}{l}501 \text { u.m. } \\
\text { Besch. }\end{array}$ & $\begin{array}{c}\text { Insgesamt } \\
\text { (ab 5 Besch.) }\end{array}$ \\
\hline & & & Westd & tschland & & \\
\hline Betriebe mit $B R$ & 6 & 44 & 66 & 77 & 88 & 10 \\
\hline Beschäftigte mit BR & 10 & 45 & 66 & 78 & 92 & 46 \\
\hline & & & Ostde & schland & & \\
\hline Betriebe mit BR & 7 & 43 & 58 & 71 & 82 & 10 \\
\hline Beschäftigte mit BR & 11 & 44 & 60 & 71 & 86 & 38 \\
\hline $\begin{array}{l}\text { *Basis: privatwirtschaftlic } \\
\text { Erwerbszweck. }\end{array}$ & riebe ab $5 \mathrm{~B}$ & ftigte, ohn & ndwirtschaft 4 & Organisatior & ohne & TCFH H \\
\hline Quelle: IAB-Betriebspane & & & & & & TTEILUNGEN \\
\hline
\end{tabular}

der Beschäftigten in den entsprechenden Betrieben ist für die letzten vier Jahre zumindest für Westdeutschland ein nomineller Rückgang unterhalb des langjährigen Niveaus feststellbar. Auch unter Berücksichtigung der veränderten Fragestellung im IAB-Betriebspanel seit 2003, durch die die Anteilswerte um ca. einen Prozentpunkt nach unten korrigiert wurden, ${ }^{1}$ ist mittlerweile doch von einem leichten Abwärtstrend im auf Beschäftigte bezogenen Deckungsgrad auszugehen. In Ostdeutschland zeigt sich die Reichweite der betrieblichen Interessenvertretung weiterhin stabil, wenngleich auf deutlich niedrigerem $\mathrm{Ni}$ veau.

Die hinlänglich bekannten größenspezifischen Unterschiede bei der Verbreitung von Betriebsräten und bei dem Anteil der betroffenen Beschäftigten zeigen sich auch in der aktuellen Befragung wieder (Tabelle 4). Nur knapp jeder 17. Kleinbetrieb (bis 50 Beschäftigte) verfügt über einen Betriebsrat. Ab 200 Beschäftigte (Freistellungsgrenze) erreicht der Anteil aber schon knapp $80 \%$ in Westdeutschland, in Ostdeutschland sind es fast drei von vier Betrieben. Prägt in betrieblicher Perspektive die große Zahl der Kleinbetriebe das Gesamtbild, so relativiert sich deren Gewicht natürlich bei Betrachtung des auf Beschäftigte bezogenen Deckungsgrads (Tabelle 3). Die geringere Verbreitung von Betriebsräten im mittel- und großbetrieblichen Segment Ostdeutschlands manifestiert sich in der dort rund acht Prozentpunkte niedrigeren Reichweite der betrieblichen Mitbestimmung.

Ebenso bestätigen sich die erwarteten starken Unterschiede im Deckungsgrad in einzelnen Branchen und das sowohl in Betriebs- als auch Beschäftigtenperspektive (ohne Tabelle). Nach wie vor besteht die größte quantitative Reichweite im traditionell stark mitbestimmten Bereich Bergbau/Energie- und Wasserversorgung, im Kredit- und Versicherungsgewerbe, in der Produktionsgüterindustrie und im Bereich Verkehr/Nachrichtenübermittlung. Das untere Ende bilden wie gewohnt die vorwiegend kleinbetrieblich strukturierten Branchen des Dienstleitungsbereichs, des Handels und der Bauwirtschaft.

\footnotetext{
1 Ellguth, P./Kohaut, S. (2005): Tarifbindung und betriebliche Interessenvertretung: Aktuelle Ergebnisse aus dem IAB-Betriebspanel, in: WSI-Mitteilungen 7, S. 398-403
} 


\section{DAS ZUSAMMENSPIEL VON BETRIEBLICHER UND ÜBERBETRIEB- LICHER INTERESSENVERTRETUNG}

In der aktuellen politischen Diskussion ist das Verhältnis von tarifvertraglicher und betrieblicher Regulierung weiterhin virulent. Vor diesem Hintergrund soll abschließend das Zusammenspiel von betrieblicher und überbetrieblicher Interessenvertretung kurz betrachtet werden.

Wie von den aktuellen Zahlen bestätigt, ist nur eine Minderheit der in der Privatwirtschaft (ab fünf Beschäftigte) beschäftigten Arbeitnehmer in Betrieben tätig, die zur Kernzone des dualen Systems der Interessenvertretung zu zählen sind. Diese Zone umfasst ein knappes Drittel der Beschäftigten in Westdeutschland und nur etwa ein Fünftel in Ostdeutschland (Tabelle 5). Bemerkenswert ist die mit $10 \%$ relativ große Bedeutung von Betrieben mit Haustarifen und einem Betriebsrat in den neuen Bundesländern. Von einer betrieblichen Vertretungslücke (Betrieb ist an einen Branchentarif gebunden, verfügt aber über keinen Betriebsrat) ist fast jeder vierte Beschäftigte in Westdeutschland, aber nur jeder sechste in Ostdeutschland betroffen. Diese vermeintlich günstigere Situation in den neuen Bundesländern ist allerdings nur der dort geringeren Reichweite der Tarifbindung geschuldet, die sich in deutlicher ausgeprägten „weißen Flecken der Tarif- und Mitbestimmungslandschaft " äußert. Während in Westdeutschland „nur“ $30 \%$ der Beschäftigten ohne Branchentarif und ohne Betriebsrat auskommen müssen, sind dies in Ostdeutschland $42 \%$.

Um das Bild etwas zu erweitern und mögliche branchenspezifische Unterschiede hinsichtlich der beschriebenen „Problemzonen" $z u$ identifizieren, sind die Ergebnisse zur Reichweite von Tarifverträgen und betrieblicher Mitbestimmung in $\mathrm{Ta}$ belle 5 zusätzlich für das Verarbeitende Gewerbe und den Dienstleistungsbereich dargestellt. Für Westdeutschland lässt sich fest-

\begin{tabular}{|c|c|c|c|c|c|c|}
\hline & \multicolumn{3}{|c|}{ Westdeutschland } & \multicolumn{3}{|c|}{ Ostdeutschland } \\
\hline & $\begin{array}{c}\text { Privat } \\
\text { wirtschaft }\end{array}$ & $\begin{array}{l}\text { daru } \\
\text { Verarb. } \\
\text { Gewerbe }\end{array}$ & $\begin{array}{l}\text { ter: } \\
\text { Dienst- } \\
\text { leistungen }\end{array}$ & $\begin{array}{l}\text { Privat- } \\
\text { wirtschaft }\end{array}$ & $\begin{array}{c}\text { daru } \\
\text { Verarb. } \\
\text { Gewerbe }\end{array}$ & $\begin{array}{l}\text { nter: } \\
\text { Dienst- } \\
\text { leistungen }\end{array}$ \\
\hline BR und Branchentarif & 31 & 48 & 24 & 19 & 21 & 20 \\
\hline BR und Haustarif & 7 & 10 & 5 & 10 & 10 & 9 \\
\hline BR und kein Tarif & 7 & 10 & 8 & 9 & 16 & 10 \\
\hline Branchentarif und kein BR & 23 & 11 & 24 & 16 & 7 & 17 \\
\hline Haustarif und kein BR & 1 & 0 & 2 & 4 & 5 & 5 \\
\hline Kein Tarif und kein BR & 30 & 20 & 37 & 42 & 41 & 39 \\
\hline Gesamt & 100 & 100 & 100 & 100 & 100 & 100 \\
\hline $\begin{array}{l}\text { Beschäftigte in Betrieben } \\
\text { mit Branchentarifvertrag }\end{array}$ & 54 & 59 & 47 & 35 & 29 & 37 \\
\hline $\begin{array}{l}\text { Beschäftigte in Betrieben } \\
\text { mit Betriebsrat }\end{array}$ & 46 & 68 & 37 & 38 & 47 & 39 \\
\hline \multicolumn{7}{|c|}{$\begin{array}{l}\text { *Basis: privatwirtschaftliche Betriebe ab } 5 \text { Beschäftigte, ohne Landwirtschaft und Organisationen ohne } \\
\text { Erwerbszweck. }\end{array}$} \\
\hline \multicolumn{7}{|c|}{$\begin{array}{l}\text { Quelle: IAB-Betriebspanel } 2006 \text { (Abweichungen von } 100 \% \text { aufgrund von Rundungsfehlern } \\
\text { möglich). }\end{array}$} \\
\hline
\end{tabular}

stellen, dass die Kernzone des dualen Systems im Verarbeitenden Gewerbe knapp die Hälfte der Beschäftigten umfasst und damit weit über dem Durchschnitt der Privatwirtschaft liegt. Mit nur jedem vierten Arbeitnehmer fällt dagegen der Dienstleistungsbereich deutlich ab. Hier haben auch die betrieblichen Vertretungslücken (24\%), und vor allem die ,weißen Flecken“ (37\%), einen ganz beträchtlichen Umfang. Im Verarbeitenden Gewerbe bleiben diese „Problemzonen" dagegen in deutlich engeren Grenzen.

In Ostdeutschland ergibt sich ein etwas anderes Bild. Hier unterscheidet sich das Verarbeitende Gewerbe weit weniger vom Dienstleistungsbereich als das in den alten Bundesländern der Fall ist. Die Kernzone der Interessenvertretung ist mit 21\% nicht größer als im Dienstleistungsbereich (20\%) und wesentlich kleiner im Vergleich zum Verarbeitenden Gewerbe Westdeutschlands (48\%). Bemerkenswert sind darüber hinaus vor allem die mit $41 \%$ beachtlichen und dem Durchschnitt der Privatwirtschaft (ebenso dem Dienstleistungsbereich) entsprechenden weißen Flecken in der Tarif- und Mitbestimmungslandschaft. Offensichtlich ist das Verar- beitende Gewerbe Ostdeutschlands nicht im gleichen überdurchschnittlichen Maße mit den beiden Institutionen der Interessenvertretung ausgestattet, wie das traditionell im Westen in diesem Wirtschaftszweig der Fall ist. Für den Dienstleistungsbereich ergibt sich hingegen in den neuen Bundesländern ein in Relation weit weniger negatives Bild als in Westdeutschland.

Ein Vergleich dieser Zahlen mit denen der Vorjahre (ohne Tabelle) zeigt für die Privatwirtschaft Westdeutschlands einen Rückgang der Kernzone der Interessenvertretung (minus vier Prozentpunkte), der vor allem durch die rückläufige Entwicklung der Flächentarifbindung im Verarbeitenden Gewerbe verursacht ist. Im Dienstleistungsbereich scheint sich das Segment, in dem sowohl ein Branchentarif als auch ein Betriebsrat besteht, eher wieder auszudehnen, allerdings von einem deutlich geringeren Niveau aus. Für Ostdeutschland bestätigt sich für die letzten Jahre auch in der gemeinsamen Betrachtung von betrieblicher und überbetrieblicher Interessenvertretung das Bild weitgehender Stabilität. 